\title{
Consideration of Gyroscopic Effect in Fault Detection and Isolation for Unbalance Excited Rotor Systems
}

\author{
Zhentao Wang, ${ }^{1}$ Arne Wahrburg, ${ }^{2}$ and Stephan Rinderknecht ${ }^{1}$ \\ ${ }^{1}$ Institute for Mechatronic Systems in Mechanical Engineering, TU Darmstadt, Petersenstr. 30, 64287 Darmstadt, Germany \\ ${ }^{2}$ Control Theory and Robotics Lab, Institute of Automatic Control and Mechatronics, TU Darmstadt, Landgraf-Georg-Str. 4, \\ 64283 Darmstadt, Germany
}

Correspondence should be addressed to Zhentao Wang, wang@ims.tu-darmstadt.de

Received 29 June 2012; Revised 1 October 2012; Accepted 11 October 2012

Academic Editor: Andrea Vania

Copyright (C) 2012 Zhentao Wang et al. This is an open access article distributed under the Creative Commons Attribution License, which permits unrestricted use, distribution, and reproduction in any medium, provided the original work is properly cited.

\begin{abstract}
Fault detection and isolation (FDI) in rotor systems often faces the problem that the system dynamics is dependent on the rotor rotary frequency because of the gyroscopic effect. In unbalance excited rotor systems, the continuously distributed unbalances are hard to be determined or estimated accurately. The unbalance forces as disturbances make fault detection more complicated. The aim of this paper is to develop linear time invariant (LTI) FDI methods (i.e., with constant parameters) for rotor systems under consideration of gyroscopic effect and disturbances. Two approaches to describe the gyroscopic effect, that is, as unknown inputs and as model uncertainties, are investigated. Based on these two approaches, FDI methods are developed and the results are compared regarding the resulting FDI performances. Results are obtained by the application in a rotor test rig. Restrictions for the application of these methods are discussed.
\end{abstract}

\section{Introduction}

Model based fault detection and isolation (FDI) methods often offer better performance than signal based FDI methods (see, e.g., [1]). Thus model based methods are widely used in the technical processes when high system performance and reliability are demanded. In ideal cases, the systems are free of disturbances or the disturbances working on the systems are known and accurate models of the systems are available for the model based FDI processes. Disturbances and model inaccuracies or uncertainties often have strong impact on FDI performance and can cause false alarms or decreased fault detection rate.

In rotor systems the unbalance forces are major disturbances for the FDI process. Although approaches for unbalance identification $[2,3]$ or balancing without trial weights $[4,5]$ are often applied to estimate modal unbalances or unbalances at the knots of the finite element model (FEM), a physical model with high accuracy and enough number of sensors are required to achieve an accurate estimation result. Actually if an elastic rotor is considered as a continuous system with infinite degrees of freedom (DOF), the continuously distributed unbalances cannot be detected to full extent. In real systems the unbalances cannot be fully determined or compensated.

In case of rotor systems with large disks, the gyroscopic effect cannot be neglected. The system behavior is then dependent on the rotor rotary frequency. If the gyroscopic matrix is known and rotor rotary frequency is measured, a parametric model dependent on rotary frequency can be built $[6,7]$ and a rotary frequency dependent FDI method can be applied. However if a rotor is not running at constant rotary frequency or if the measurement of the rotary frequency is not accurate enough, the rotary frequency dependent fault detection filter is often not applicable. Also an accurate parametric model is often not available for model based FDI.

In contrast to the rotary frequency dependent design, linear time invariant (LTI) fault detection filters with simple and easy to implement structures are developed in this paper. Therefore, we consider a constant model at a certain rotary frequency as a nominal model and the gyroscopic effect is formulated as model uncertainties or unknown inputs. The FDI problems for rotor systems become then FDI problems 
with disturbances and model uncertainties or unknown inputs. To cope with these effects, robustness of the designed fault detection filters is crucial for a reliable performance.

Robust FDI methods are mostly based on two approaches: decoupling of unknown inputs [8] and optimization by means of different objective functions [9].

The idea of unknown inputs was firstly introduced by Watanabe and Himmelblau [10] with the design of unknown input observer (UIO) to decouple the influences of unknown inputs on the residuals. Unknown inputs summarize the influences of unknown disturbances with a certain distribution on the plant. In some cases, model uncertainties can be considered as unknown inputs with determined distribution $[11,12]$, thus by decoupling of the unknown inputs the influences of model uncertainties are also decoupled. Besides UIO, another observer based method can be found, for example, in $[13,14]$ which utilizes eigenstructure assignment of the observer and a residual weighting matrix. Parity equations based methods to decouple unknown inputs can be found, for example, in [1] or [9]. In the last years, least order fault detection filters based on null space design are investigated $[15,16]$. Methods that account for sinusoidal unknown inputs, which is the case of rotor system, can be found in [17].

Through the development of techniques to solve linear matrix inequalities (LMIs) [18], optimization methods are intensively investigated in FDI processes in the last years. Uncertainties in the system matrices can be recast as disturbances [19] or treated directly [20]. Some publications focus on ensuring sufficient disturbance attenuation and fault sensitivity and at the same time rejecting the influence of control inputs onto the residuals (see, e.g., [21, 22]). Other publications rely on matching the input-output behavior of the overall system consisting of plant and residual generator to a given reference model $[23,24]$.

A lot of researches on FDI in rotor systems are done in the last decades. Some of the researches focus on the fault characteristic [25-27] and others test different FDI methods in rotor systems [28-30]. The gyroscopic effect is often not of concern in the FDI processes. Despite other aspects to investigate symptoms of fault appearances in rotor systems (e.g., special orbit or frequency domain analysis), this paper is mainly focusing on developing general FDI methods dealing with gyroscopic effect and disturbances (e.g., from initial unbalances). The objective is to detect input faults (e.g., changes of unbalances), which are not simply distinguishable from disturbances under the consideration of the gyroscopic effect. To this end, two different approaches to describe the gyroscopic effect are considered. On the one hand, the effect is formulated as unknown inputs. On the other hand, it is interpreted as uncertainties in the system model. Based on these two descriptions, different approaches to design fault detection filters are investigated. In Section 2 we present the test rig on which we implement and test the obtained fault detection filters. We consider both physical models and identified models that are used for the filter design and the modeling of the gyroscopic effect. Section 3 briefly summarizes the method to estimate the unknown input distribution matrix and the different design approaches for fault detection filters which can be employed for rotor systems. Mainly, an unknown input observer and model matching approaches are considered. In Section 4, we test the feasibility of these two approaches by means of simulation using the test rig model. The application results of the methods on the test rig are presented in Section 5 before a conclusion is given.

1.1. Notational Aspects. With the symbol $I$, we abbreviate an identity matrix, while 0 denotes a matrix of zeros of appropriate dimensions. For a matrix $M \in \mathbb{R}^{n \times m}$, the singular values are written as $\sigma_{1}, \sigma_{2}, \ldots, \sigma_{p}$, with $p=\min \{n, m\}$. For a stable transfer matrix $G(s)$, the $H_{\infty}$-norm is the maximum singular value of $G(j \omega)$ over all frequencies and abbreviated as $\sup _{\omega \in[0, \infty)} \max _{i=1, \ldots, p} \sigma_{i}(G(j \omega))=\|G(s)\|_{\infty}$. For a square and symmetric matrix $P$, positive (negative) definiteness is denoted by $\succ(\prec)$. Elements of such matrices, which are readily inferred by symmetry, are abbreviated by the symbol $\star$. Furthermore, we write $\operatorname{He}(Q)=Q+Q^{\top}$ for the symmetric part of a matrix $Q$.

\section{Modeling}

2.1. Test Rig. The test of the FDI methods and the comparison of their performances are carried out on a rotor test rig presented in Figure 1.

This test rig is constructed at the Technische Universität Darmstadt to test different control strategies and FDI methods in rotor systems under consideration of gyroscopic effect. The flexible shaft has a diameter of $9 \mathrm{~mm}$ and $320 \mathrm{~mm}$ in length. Two discs with relatively large inertia are mounted on the shaft unsymmetrically to enhance the gyroscopic effect. The gyroscopic effect can be observed from the Campbell diagram of the test rig in Figure 2. The test rig is supported by an active bearing on the left side and a passive bearing on the right side. The active bearing is equipped with two piezoelectric stack actuators with maximum strokes of $60 \mu \mathrm{m}$. The actuators are arranged orthogonal to each other. Against each piezoelectric actuator a preload spring is mounted, so that the piezoelectric elements only work under pressure forces. Four displacement sensors are implemented in the test rig on 2 sensor planes along the rotor in pairs (see Figure 1). On every sensor plane, the sensors are arranged in the same directions as the actuators. The first 2 eigenfrequencies of non-rotating rotor are about $39 \mathrm{~Hz}$ and $97 \mathrm{~Hz}$. The first and second eigenforms are presented in Figure 3. The rotor is driven by a 250 watt DC motor with maximal rotation speed of $1000 \mathrm{rad} / \mathrm{s}$ and runs through 2 resonances at $47 \mathrm{~Hz}(295 \mathrm{rad} / \mathrm{s})$ and $108 \mathrm{~Hz}(678 \mathrm{rad} / \mathrm{s})$.

A finite element model is built for the test rig on the basis of Timoshenko beam theory [31]. The bearings are modeled using discrete stiffness, inertia, and piezoelectric elements. The active bearing is coupled with the shaft by applying the forces of the piezoelectric actuators to the shaft which are dependent on the strains of the actuators and the voltages applied to the actuators. The strains of the actuators are calculated from the vibration of the shaft. The model of the piezoelectric actuators obeys the law 

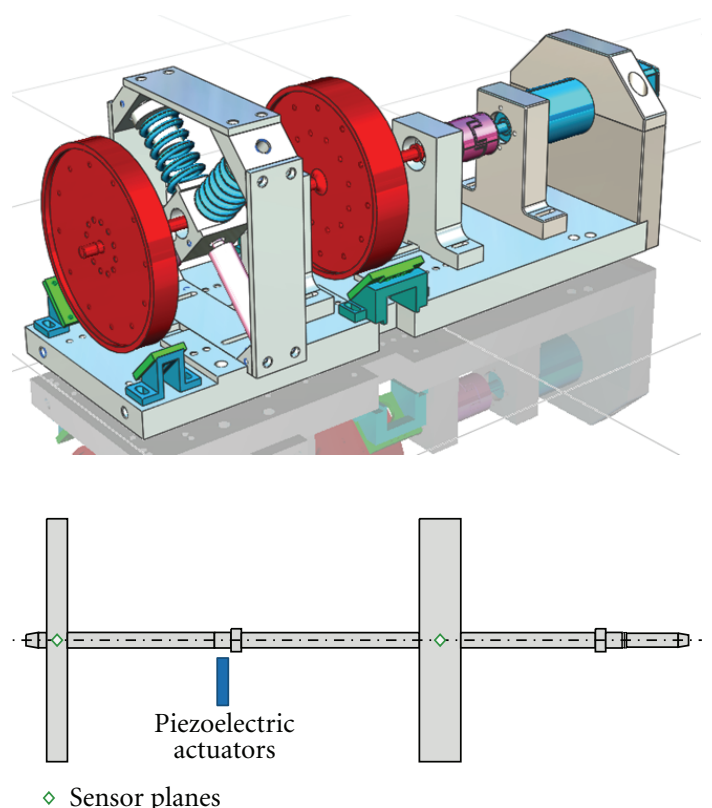

Figure 1: Configuration of the rotor test rig.

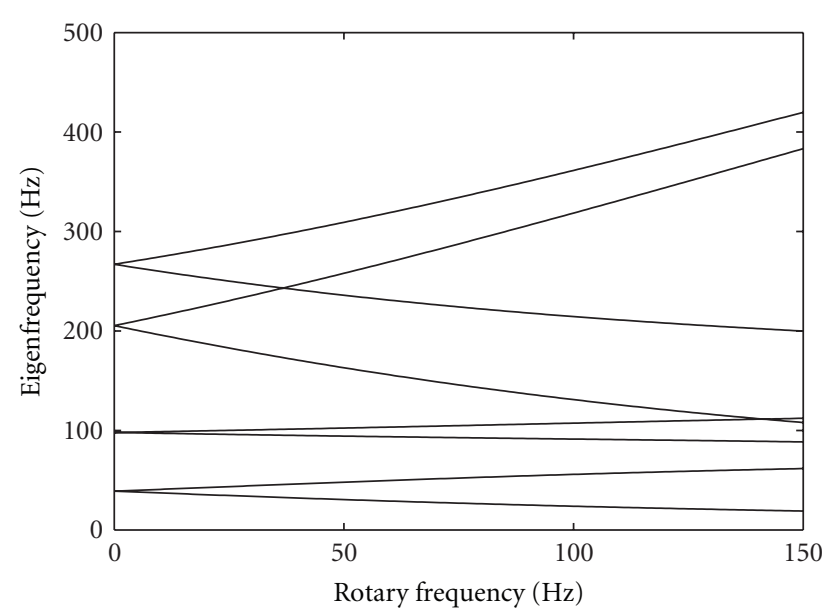

FIgURE 2: Campbell diagram of the rotor system.

of piezoelectric material. The piezoelectric actuators are used for the vibration control. The FDI methods are not influenced by the actuators. They are thus not considered in the FDI process. The damping of the rotor system is considered as viscous damping. The damping ratio is set to $1 \%$, in order to match the measured frequency responses of the test rig. For the implementation of FDI methods, the finite element model is reduced by means of modal reduction technique. A low order model with 8 modal degrees of freedom (DOF), that is, $16 \mathrm{DOF}$ in the state space representation, is proven to be accurate enough for the relevant frequency range. The finite element model of the rotor systems is proper, thus the feed through part does not

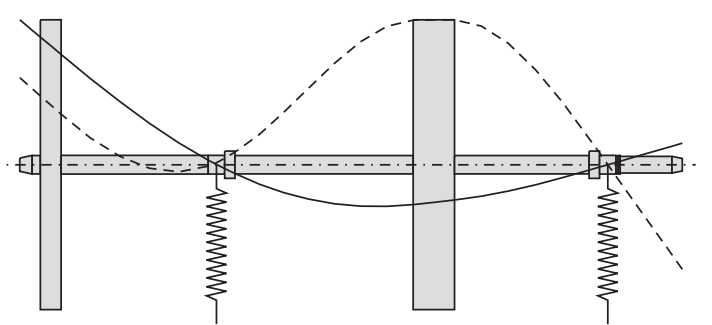

Figure 3: The first and second eigenforms of the rotor test rig.

exist in the state space representation. The state space model of the rotor is given in

$$
\begin{gathered}
\dot{x}=A_{G}(\Omega) x+B u+E_{D} d_{D}, \\
y=C x,
\end{gathered}
$$

where $x$ is the vector of system states, $u$ is the control input vector, that is, the voltages applied to the actuators, $d_{D}$ describes the disturbances, that is, forces (e.g., generated by initial unbalances) acting on the shaft, and $y$ are the system outputs, that is, the sensor signals. $A_{G}(\Omega), B, C, E_{D}$ are system matrices with appropriate dimensions, where $A_{G}(\Omega)$ is dependent on the rotor rotary frequency $\Omega$ because of gyroscopic effect. The initial unbalances are modeled as unbalances randomly distributed in both axial and circumferential directions of rotor shaft. The masses and eccentricities of initial unbalances are randomly chosen with strongly weighted unbalances on the rotor discs. The model (1a) and (1b) is controllable and observable for whole rotary frequency range.

2.2. Model for FDI Process. Since multiplicative faults can be transformed in an additive way [9], the faults are modeled as additive faults generally. In rotor systems the influences of unbalances and gyroscopic effect (as will be presented in Section 2.3) are periodical with the rotor rotary frequency. The major focus of this work is to detect input faults (e.g., rotor disc break) that is influenced by the gyroscopic effect and periodical with the rotor rotary frequency. These faults are not simply distinguishable from the influences of gyroscopic effect and disturbances from initial unbalances. Output faults (i.e., sensor faults) are not influenced by the gyroscopic effect and are normally not periodical. Mostly, they can be easily detected in the frequency domain. Thus the output faults are not of the interest in this paper and are not explicitly considered in the following. But the methods introduced in this paper are also applicable for output faults.

Some limitations of the model and different situations are considered in the FDI process according to the knowledge of the rotor system

(i) The initial unbalances cannot be detected to full extent, thus the disturbance term $E_{D} d_{D}$ in (1a) and (1b) is supposed to be unknown.

(ii) If enough physical information about the rotor system is available, the system can be modeled using 
a physical model. On the basis of the model of nonrotating rotor (i.e., setting $A=A_{G}(0)$ in model (1a) and (1b)), the gyroscopic effect can be represented as an additive term $\Omega A_{g}$ to the $A$ matrix. The available model in this case is thus

$$
\begin{gathered}
\dot{x}=\left(A+\Omega A_{g}\right) x+B u+F f, \\
y=C x,
\end{gathered}
$$

where $f$ represents considered faults and $F$ is its input matrix.

(iii) If a physical model cannot be build or accuracy of a physical model is insufficient, identified model can be used. In this case, the knowledge about gyroscopic matrix $A_{g}$ is supposed to be unknown. Only identified models at certain rotor rotary frequencies are supposed to be available. If the gyroscopic effect is considered as model uncertainties, identified models at different rotary frequencies $\Omega_{n}$

$$
\begin{gathered}
\dot{x}=A_{\Omega n} x+B u+F f, \\
y=C x,
\end{gathered}
$$

with $A_{\Omega n}=A_{G}\left(\Omega_{n}\right)$ are required, so that different situations of the systems can be considered in the FDI process. For the design based on the model matching approach (see Sections 3.3 and 4.2), $A_{n}=$ $A_{G}\left(1 / 2 \Omega_{\max }\right)$ is used as a nominal model, where $\Omega_{\max }$ is the maximum relevant rotor rotary frequency. Therewith, the distance of models for $\Omega=0$ and $\Omega=$ $\Omega_{\max }$ to the nominal model is the same with respect to the parameter $\Omega$. This facilitates the solution of the model matching problem. If the gyroscopic effect is described as unknown inputs, the model of nonrotating rotor

$$
\begin{gathered}
\dot{x}=A x+B u+F f, \\
y=C x,
\end{gathered}
$$

with $A=A_{G}(0)$ is used as nominal model for the FDI process. Thereby, the gyroscopic effect has not to be considered in the nominal model (5a) and (5b).

2.3. Consideration of Initial Unbalances and Gyroscopic Effect. In order to achieve robustness against disturbances (e.g., from initial unbalances), information about the disturbances is required. The way that unbalance forces influence the rotor system can be determined by means of measurements on the test rig [32]. Detection of the real unbalance distribution is not needed. If unknown inputs $d$ and their distribution matrix $E$ are used to represent the influences of initial unbalances, the system model for FDI is extended as

$$
\begin{gathered}
\dot{x}=\left(A+\Omega A_{g}\right) x+B u+E d+F f, \\
y=C x,
\end{gathered}
$$

in case of physical model and

$$
\begin{gathered}
\dot{x}=A_{\Omega n} x+B u+E d+F f, \\
y=C x,
\end{gathered}
$$

in case of identified model. Ideally, the unknown inputs $d$ have the same influences via their distribution matrix $E$ as initial unbalances. For FDI process only the distribution matrix $E$ is determined, the unknown inputs $d$ are not detected. Note that the estimated matrix $E$ is generally not identical and does not necessarily to be equal to or even have the same dimension as the real input matrix of disturbances $E_{D}$.

In rotor dynamics, conservation of angular momentum leads to a coupling between the rotational degrees of freedom in a rotating shaft. The effect of the coupling on rotor discs can be considered as moments generated by the discs and acting periodically on the rotor shaft. The effects of coupling of shaft elements are normally small and negligible. For the modeling, the rotor system can be regarded as a simple rotor system without gyroscopic effect (i.e., model (5a) and (5b)) plus disturbance moments representing gyroscopic effect. The influences of gyroscopic effect and initial unbalances can be represented together as unknown inputs $d$ generally with their distribution matrix E. By means of measurements on the test rig at different rotary frequencies, the matrix $E$ can be determined [33]. On the basis of model (5a) and (5b), the model for FDI is extended as

$$
\begin{gathered}
\dot{x}=A x+B u+E d+F f, \\
y=C x .
\end{gathered}
$$

\section{Theory}

Based on the model (6a) and (6b), (7a) and (7b), or (8a) and (8b), three problems have to be solved to achieve robustness against gyroscopic effect and influences of unbalances in FDI processes in rotor systems.

(1) Represent influences of unbalances and in case of model (8a) and (8b) also the gyroscopic effect using unknown inputs.

(2) Design FDI scheme which is robust against unknown inputs.

(3) Design FDI scheme which is robust against model uncertainties if model (6a) and (6b) or (7a) and (7b) is used.

3.1. Estimation of Unknown Input Distribution Matrix. Dependent on the knowledge of the disturbances, model inaccuracies, or model uncertainties, different methods [3436] can be used to determine the unknown input distribution matrix. Without any knowledge about the initial unbalances and gyroscopic effect (in case of model (8a) and (8b)), methods using measured signals (e.g., deconvolution method or augmented observer) are suitable for the estimation in rotor systems. Measurements for the estimation of the unknown 
input distribution matrix are simulated using test rig model (1a) and (1b) in this paper. The active bearing is not activated (i.e., $u=0$ ) for the estimation of unknown input distribution matrix and the rotor is only excited by initial unbalances.

3.1.1. Deconvolution Method. The de-convolution method introduced in $[8,35]$ is based on a discrete state space model of the system:

$$
\begin{gathered}
x(k+1)=A_{\mathrm{disc}} x(k)+B_{\mathrm{disc}} u(k)+E_{\mathrm{disc}} d(k), \\
y(k)=C_{\mathrm{disc}} x(k),
\end{gathered}
$$

where $A_{\text {dics }}, B_{\text {dics }}$, and $C_{\text {dics }}$ are the system matrices of the discrete time model. The term $E_{\text {disc }} d(k)$ represents unknown inputs with the distribution matrix. If the control inputs are set to zero, the difference between measured output $y_{M}(k)$ and calculated model response $y(k)$, that is, the residual $r(k)$, with unknown initial condition $x(0)$ can be described as:

$$
\begin{aligned}
r(k)= & y_{M}(k)-y(k) \\
= & y_{M}(k)-C_{\mathrm{disc}} A_{\mathrm{disc}}^{k} x(0) \\
& -\sum_{i=1}^{k} C_{\mathrm{disc}} A_{\mathrm{disc}}^{i-1} \underbrace{E_{\mathrm{disc}} d(k-i)}_{d_{1}(k-i)} .
\end{aligned}
$$

For every time step $k$, a disturbance vector

$$
d_{1}(k-1)=E_{\mathrm{disc}} d(k-1),
$$

is obtained by solving $r(k)=0$ :

$$
\begin{aligned}
C_{\mathrm{disc}} d_{1}(k-1)= & y_{M}(k)-C_{\mathrm{disc}} A_{\mathrm{disc}}^{k} x(0) \\
& -\sum_{i=2}^{k} C_{\mathrm{disc}} A_{\mathrm{disc}}^{i-1} d_{1}(k-i) .
\end{aligned}
$$

If matrix $A_{\text {disc }}$ is stable, $\lim _{l \rightarrow \infty} A_{\text {disc }}^{l}=0$. The unknown initial condition can be set to $x(0)=0$, the error caused by $x(0)$ will decay in some time steps. In (13) if and only if $\operatorname{rank}\left(C_{\text {disc }}\right)=\operatorname{dim}(x)$ a single solution can be calculated. If less linearly independent measurements than the model order are available (i.e., $\left.\operatorname{rank}\left(C_{\text {disc }}\right)<\operatorname{dim}(x)\right)$, (13) is underdetermined and $d_{1}(k)$ has to be estimated under other restrictions (e.g., assume that only part of the eigenforms are exited). Thus the number of linearly independent measurements (equals $\operatorname{rank}\left(C_{\text {disc }}\right)$ ) is crucial for the accuracy of the estimation.

For a measurement with $k_{\max }$ time steps, a vector set

$$
M=\left[d_{1}(1), d_{1}(2), \ldots d_{1}\left(k_{\max }\right)\right]
$$

with $k_{\max }$ elements can be calculated. The matrix $E_{\text {disc }}$ can be considered as a set of vectors and the elements of $d(k)$ as weighting factors in (12). The matrix $M$ spans a space in which all the vectors of $E_{\text {disc }}$ lie. The matrix $E_{\text {disc }}$ can be calculated on the basis of singular value decomposition of $M$ [8]:

$$
M=U\left[\operatorname{diag}\left(\sigma_{1}, \sigma_{2}, \ldots, \sigma_{p}\right) 0\right] V^{T}
$$

where matrices $U, V$ are left and right singular matrices and $\sigma_{1}, \sigma_{2}, \ldots, \sigma_{p}$ are the singular values with $\sigma_{1} \geq \sigma_{2} \geq \cdots \geq \sigma_{p}$. The matrix $E_{\text {disc }}$ is obtained by selecting a few of the most significant singular values:

$$
E_{\text {disc }}=U\left[\operatorname{diag}\left(\sigma_{1}, \sigma_{2}, \ldots, \sigma_{q}\right)\right],
$$

where $\sigma_{q+1}, \sigma_{q+2} \cdots \ll \sigma_{1}$ can be neglected. The unknown input distribution matrix in the continuous time model is obtained by transforming $E_{\text {disc }}$ into continuous time.

3.1.2. Augmented Observer. Since the unknown input signals in rotor systems are sinusoidal, the vector $d_{1}$ in (12) can be directly estimated using an augmented observer on the basis of an augmented system model for sinusoidal disturbances:

$$
\begin{gathered}
{\left[\begin{array}{c}
\dot{x} \\
\dot{x}_{d}
\end{array}\right]=\left[\begin{array}{cc}
A & H_{d} \\
0 & A_{d}
\end{array}\right]\left[\begin{array}{c}
x \\
x_{d}
\end{array}\right]+\left[\begin{array}{c}
B \\
0
\end{array}\right] u,} \\
y=\left[\begin{array}{ll}
C & 0
\end{array}\right]\left[\begin{array}{c}
x \\
x_{d}
\end{array}\right] .
\end{gathered}
$$

The augmented part of the system matrix is from the disturbance model:

$$
\begin{aligned}
& \dot{x}_{d}=A_{d} x_{d}, \\
& d_{1}=C_{d} x_{d},
\end{aligned}
$$

which describes the sinusoidal signals as a differential equation in state space form. Determination of the system matrices can be found in [17]. The vector $d_{1}$ can be directly observed, for example, by constructing a Lueberger observer on the basis of augmented model (17a) and (17b).

3.2. Robust Fault Detection against Unknown Inputs. Robust FDI methods against unknown inputs are normally based on the idea of decoupling the influences of unknown inputs on the residuals or optimizing the objective to attenuate unknown inputs and enhance fault sensitivity on the residuals.

3.2.1. Fault Detection by Means of Decoupling Unknown Inputs. In the last decades, different methods to decouple unknown inputs have been developed. Among others, unknown input observer (UIO) [8] is one of the most used methods. UIO takes the structure of the general form of Luenberger observer (see Figure 4):

$$
\begin{gathered}
\dot{z}=F z+T B u+K_{\mathrm{UIO}} y, \\
\hat{x}=z+H y,
\end{gathered}
$$




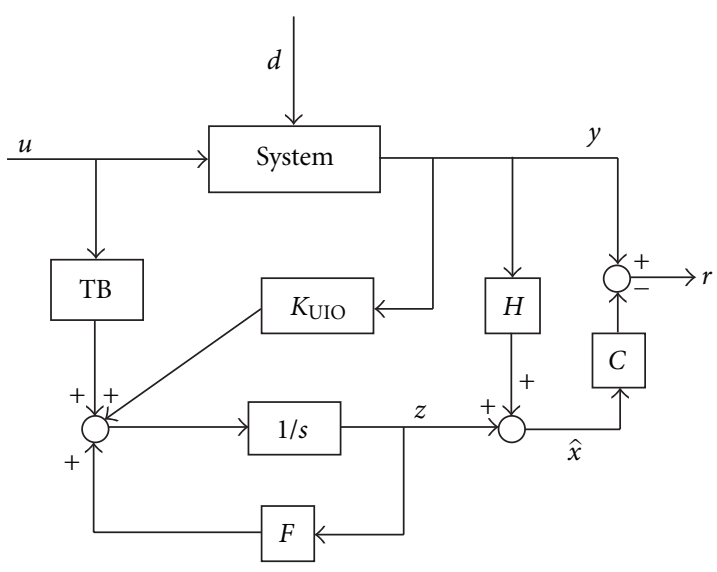

Figure 4: Structure of unknown input observer.

where $\hat{x}$ is an estimate of $x$. If

$$
\begin{gathered}
(I-H C) E=0, \\
T=I-H C, \\
F=T A-K_{1} C, \\
K_{\mathrm{UIO}}=K_{1}+F H,
\end{gathered}
$$

the estimation error, $e=x-\hat{x}$, can be described by

$$
\dot{e}=F e .
$$

If the UIO in form of (19) and (20) is stable, the estimation error will go to zero and the influence of unknown inputs is then decoupled on the residual $r=\mathrm{Ce}$. The matrix $K_{1}$ is chosen to stabilize the UIO and give $e$ desired dynamics, for example, by pole placement of the system $\left((T A)^{T}, C^{T}\right)$.

It is pointed out in [8], that a UIO in form of (19) and (20) exist if and only if: $\operatorname{rank}(C E)=\operatorname{rank}(E)$ and $(C, T A)$ is a detectable pair. This indicates that the maximum number of disturbances considered cannot be larger than the number of independent measurements if the disturbance input matrix $E$ is of full column rank.

Other methods using observer structures $[13,14]$ or parity equations $[15,16]$ are based on the idea to design residual spaces that are null spaces of unknown inputs. Since the unknown inputs are sinusoidal, disturbance observers for sinusoidal disturbances $[32,33]$ can also be used for the decoupling of unknown inputs. These methods are not restricted to the condition that $(C, T A)$ is a detectable pair and some of them require less computing time. But the restriction that the number of disturbances cannot be larger than the number of linearly independent measurements is also valid for these methods $[8,9]$. The major advantage of UIO is that it is rotary frequency independent and the state vector can be correctly observed under the influences of unknown inputs. This can be useful for fault diagnosis or the implementation of state feedback based controllers.

3.2.2. Fault Detection by Means of Optimization. Besides decoupling methods, robustness against unknown inputs can also be achieved using multi-objective optimization (see $[8,9]$ for an overview). The residual generator is generally described as:

$$
r(s)=G_{r d}(s) d(s)+G_{r f}(s) f(s)
$$

where $G_{r d}(s)$ and $G_{r f}(s)$ represent the influences of unknown inputs and faults on the residual. In case of observer based fault detection,

$$
\begin{gathered}
\dot{\hat{x}}=A \hat{x}+B u+L(y-C \hat{x}), \\
r=V(y-C \hat{x})
\end{gathered}
$$

is used and the transfer matrices relating disturbances (resp., faults) and residuals can be written as:

$$
\begin{aligned}
& G_{r d}(s)=V C(s I-A+L C)^{-1} E \\
& G_{r f}(s)=V C(s I-A+L C)^{-1} F .
\end{aligned}
$$

The robustness problem is then formulated as to find matrices $L$ and $V$, such that:
(i) $A-L C$ is stable,
(ii) $\left\|G_{r d}\right\|<\gamma, \gamma>0$,
(iii) $\left\|G_{r f}\right\| \rightarrow \max$.

The operator $\|\square\|$ denotes different norms and can be chosen dependent on the system behavior. $\gamma$ is a given constant.

3.3. Robust Fault Detection with Respect to Model Uncertainties. An intuitive approach to account for uncertain system matrices is the approximative model matching technique described, for example, in $[9,24]$. The basic idea is using a reference model $G_{\text {ref }}(s)$, which characterizes the desired input-output behavior with generalized input $w=\left[\begin{array}{lll}u^{\top} & f^{\top} & d^{\top}\end{array}\right]^{\top}$ and generalized output $r_{\text {ref }}$. Then an observer of the form (27a) and (27b) is to be parameterized such that the generated residual $r$ matches $r_{\text {ref }}$ as well as possible. Notice that all exogeneous inputs are lumped into $w$. Intuitively, since the overall system consisting of plant and residual generator is to match the reference model, its selection is crucial to the quality of the resulting FDI system $[37,38]$.

Because of this, a two step procedure is proposed in [24]. Firstly, an observer parametrization $L^{*}$ and $V^{*}$ is obtained for a system without any parametric uncertainties, that is, the nominal model with system matrix $A_{n}$. This observer is designed using an optimization based approach aiming at an optimal compromise between efficient disturbance rejection and sufficient fault sensitivity for this system. The resulting input-output behavior generated by this fault detection observer is then regarded as the reference model. Following [39], the optimization problem in the first design step is

$$
\underset{L, V}{\operatorname{minimize}} \frac{\left\|G_{r d}(s)\right\|_{\infty}}{\sigma_{i}\left(G_{r f}(j \omega)\right)} \quad \forall \omega \in[0, \infty) .
$$


Therein, $\sigma_{i}\left(G_{r}(j \omega)\right)$ describes the singular values of $G_{r f}(j \omega)$. The solution is also given in [39] as

$$
\begin{gathered}
L^{*}=\left(E D_{d}^{\top}+Y C^{\top}\right) Q^{-1}, \\
V^{*}=Q^{-1 / 2},
\end{gathered}
$$

with $Q=D_{d} D_{d}^{\top}$. The matrix $D_{d}$ describes the influence of disturbances on the measured outputs. As they are not part of the model (6a) and (6b) or (7a) and (7b), we elaborate further on its choice in Section 4.2. The matrix $Y \geq 0$ is obtained by solving the algebraic Riccati equation

$$
\begin{aligned}
Y\left(A_{n}-E D_{d}^{\top} Q^{-1} C\right)^{\top} & +\left(A_{n}-E D_{d}^{\top} Q^{-1} C\right) Y-Y C^{\top} Q^{-1} C Y \\
& +\cdots+E\left(I-D_{d}^{\top} Q^{-1} D_{d}\right)^{2} E^{\top}=0 .
\end{aligned}
$$

Having obtained $L^{*}$ and $V^{*}$, we can describe the reference model with $x_{\text {ref }}=x-\hat{x}$ as

$$
\begin{gathered}
\dot{x}_{\text {ref }}=\underbrace{\left(A_{n}-L^{*} C\right)}_{A_{\text {ref }}} x_{\text {ref }}+\underbrace{F}_{B_{\text {ref }, f}} f+\underbrace{\left(E-L^{*} D_{d}\right)}_{B_{\text {ref }, d}} d, \\
r_{\text {ref }}=\underbrace{V^{*} C}_{C_{\text {ref }}} x_{\text {ref }}+\underbrace{V^{*} D_{d}}_{D_{\mathrm{ree}, d}} d .
\end{gathered}
$$

Secondly, the model matching approach minimizes the worst case deviation between the input-output behavior of the reference model and all possible plants resulting from the uncertainties. This can be formalized as

$$
\underset{L, V}{\operatorname{minimize}} \max _{\Omega}\left(\left\|G_{r w}(s, \Omega)-G_{\mathrm{ref}}(s)\right\|_{\infty}\right) \quad \forall \Omega \in\left[0, \Omega_{\max }\right],
$$

which is a model matching problem (MMP). Therein, $\Omega_{\max }$ is the highest relevant rotor rotary frequency. Therewith, following the worst-case paradigm, the largest deviation of $G_{r w}(s)$ over all parametric uncertainties with respect to the reference model is minimized in the sense of the $H_{\infty}$-norm. Notice that $G_{\text {ref }}(s)$ accounts for disturbances $d$. Neglecting the influence of $d$ in the model matching problem could lead to poor results due to an invalid reference model [24]. However, (34) implies the evaluation of the $H_{\infty}$-norm for an infinite number of system models and is thus not practicable in the given form. To overcome this, we first introduce $\tilde{x}=$ $\left[\begin{array}{lll}(x-\hat{x})^{\top} & x_{\mathrm{ref}}^{\top} & x^{\top}\end{array}\right]^{\top}$ and $\xi=r-r_{\text {ref. }}$. The input-output behavior of the transfer matrix difference in (34) is then described by

$$
\begin{gathered}
\dot{\tilde{x}}=\underbrace{\left[\begin{array}{ccc}
A_{n}-L C & 0 & \Delta A(\Omega) \\
0 & A_{\mathrm{ref}} & 0 \\
0 & 0 & A_{G}(\Omega)
\end{array}\right]}_{A_{\xi w}(\Omega)} \tilde{x}+\underbrace{\left[\begin{array}{ccc}
0 & F & E-L D_{d} \\
0 & B_{\mathrm{ref}, f} & B_{\mathrm{ref}, d} \\
B & F & E
\end{array}\right]}_{B_{\xi w}} w, \\
\xi=\underbrace{\left[\begin{array}{ll}
V C-C_{\mathrm{ref}} & 0
\end{array}\right]}_{C_{\xi w}} \tilde{x}+\underbrace{\left[\begin{array}{lll}
0 & 0 & V D_{d}-D_{\mathrm{ref}, d}
\end{array}\right]}_{D_{\xi_{w}}} w .
\end{gathered}
$$

Note that therein, $\Delta A(\Omega)=A_{G}(\Omega)-A_{n}$ is introduced as an abbreviation. Hence, $G_{\xi w}(s, \Omega)=$ $C_{\xi_{w}}\left(s I_{3 n}-A_{\xi w}(\Omega)\right)^{-1} B_{\xi_{w}}+D_{\xi_{w}}$ is the transfer matrix relating $w$ and $\xi$ and a measure for the gain from $w$ to $\xi$ is $\left\|G_{\xi w}(s, \Omega)\right\|_{\infty}$. Employing the Bounded Real Lemma [18], we can state the following.

Lemma 1. Given the stable linear time-invariant system $\dot{\tilde{x}}=$ $A_{\xi_{w}}(\Omega) \tilde{x}+B_{\xi_{w}} w, \xi=C_{\xi_{w}} \tilde{x}+D_{\xi_{w}} w$ with transfer matrix $G_{\xi w}(s, \Omega)$. Then $\left\|G_{\xi w}(s, \Omega)\right\|_{\infty}<\gamma$ holds if and only if there exists a real symmetric matrix $P \succ 0$, such that

$$
\left[\begin{array}{ccc}
\mathrm{He}\left(P A_{\xi w}(\Omega)\right) & P B_{\xi_{w}} & C_{\xi w}^{\top} \\
\star & -\gamma I & D_{\xi w}^{\top} \\
\star & \star & -\gamma I
\end{array}\right] \prec 0 .
$$

The symbol $\star$ denotes elements readily inferred by symmetry and $\mathrm{He}(Z)=Z+Z^{\top}$ is used as an abbreviation.

It is important to note that if a model matching level $\gamma$ is calculated employing a common Lyapunov matrix $P$ for a finite number of systems with $A_{\xi w}\left(\Omega_{i}\right), i=1, \ldots, N$, this $\gamma$ is true for all possible systems which lie in the convex hull spanned by $A_{\xi w}\left(\Omega_{i}\right)$. Thus, only a finite number of $N$ systems has to be considered, which makes the problem tractable.

Equation (36) is not linear because $A_{\xi w}(\Omega)$ depends on $L$. In order to obtain a convex optimization problem, we impose a blockdiagonal structure for the Lyapunov matrix as in [24]. With

$$
P=\left[\begin{array}{ccc}
P_{11} & 0 & 0 \\
\star & P_{22} & P_{23} \\
\star & \star & P_{33}
\end{array}\right]
$$

we have

$$
\begin{gathered}
\underset{P, X, V}{\operatorname{minimize}} \gamma \text { subject to } \\
P \succ 0, \\
{\left[\begin{array}{ccc}
\Xi_{11, i} & \Xi_{12} & \Xi_{13} \\
\star & -\gamma I & \Xi_{23} \\
\star & \star & -\gamma I
\end{array}\right] \prec 0, \quad i=1, \ldots, N}
\end{gathered}
$$

with

$$
\begin{aligned}
& \Xi_{11, i}=\operatorname{He}\left(\left[\begin{array}{ccc}
P_{11} A_{n}-X C & 0 & P_{11} \Delta A\left(\Omega_{i}\right) \\
0 & P_{22} A_{\text {ref }} & P_{23} A_{G}\left(\Omega_{i}\right) \\
0 & P_{23}^{\top} A_{\text {ref }} & P_{33} A_{G}\left(\Omega_{i}\right)
\end{array}\right]\right), \\
& \Xi_{12}=\left[\begin{array}{ccc}
0 & P_{11} F & P_{11} E-X D_{d} \\
P_{23} B & P_{22} B_{\mathrm{ref}, f}+P_{23} F & P_{22} B_{\mathrm{ref}, d}+P_{23} E \\
P_{33} B & P_{23}^{\top} B_{\mathrm{ref}, f}+P_{33} F & P_{23}^{\top} B_{\mathrm{ref}, d}+P_{33} E
\end{array}\right], \\
& \Xi_{13}=C_{\xi w}^{\top}, \\
& \Xi_{23}=D_{\xi w}^{\top} .
\end{aligned}
$$

Therein, a new variable $X=P_{11} L$ is introduced. Notice that (37)-(39d) is a convex optimization problem, since the objective function is convex and all constraints are formulated in terms of LMIs and are hence convex as well. After 
solving (37)-(39d), the observer matrix is reconstructed by $L=P_{11}^{-1} X$. However, it should be noticed that the structure of the Lyapunov matrix is constrained in order to render the problem convex. This introduces some conservatism to the solution. Without these restrictions, the problem includes bilinear matrix inequalities (BMIs), which are nonconvex and difficult to solve. The solution obtained by solving (37)(39d) might be enhanced using path-following techniques [40] or the approach proposed in [41].

\section{Feasibility Test of the Methods}

The feasibility test is done by means of simulation using the test rig model. As a example a unbalance change on the left rotor disc of the test rig (see Figure 1) is considered as fault. Fault detection processes based on the two different approaches presented in Sections 3.2 and 3.3 are investigated. In the first one, gyroscopic effect is considered as unknown inputs and in the second one, gyroscopic effect is considered as model uncertainties.

Investigation in the simulation shows that in order to represent the initial unbalances and gyroscopic effect for the whole rotary frequency range, a 6-column distribution matrix $E$ of unknown inputs is required. And for a small rotary frequency range a 2 -column matrix $E$ as a subspace of the 6-column matrix $E$ is enough. Under the real condition of the test rig with 4 sensors, the decoupling methods, for example, UIO are not applicable due to the conditions discussed in the Section 3. Although there are no strong restrictions of sensor number to apply the optimization methods, the number of the measurements has a strong influence on the optimization results. In order to test the feasibility of the methods for whole frequency range, we propose a better FDI condition with a 8 sensor configuration of the test rig presented in Figure 5 and test the methods by means of simulation. The 4 additional sensors are placed on 2 sensor planes, which are supposed to have the same configuration as the original sensor planes on the test rig.

The residual generation process can be generally considered as signal processing with mixed signal sources. The residuals can often be scaled arbitrarily in the parameter design and have a mixed unit, which does not have physical interpretation. UIO is a special case, whose residuals have the same units as the outputs. But for the sake of simplicity and comparison, we consider the residuals without units in general.

4.1. Consideration of Gyroscopic Effect as Unknown Inputs. For the approach to consider gyroscopic effect as unknown inputs, the model in form of $(8 \mathrm{a})$ and $(8 \mathrm{~b})$ is used. A 6column matrix $E$ is estimated to represent both influences of disturbances (initial unbalances) and gyroscopic effect. For the application of UIO, there must be at least as many linearly independent measurements as unknown inputs (see Section 3.2). The matrix $E$ is estimated with 6 columns to hold the condition and achieve a good approximation. For the estimation of the matrix $E$ the control inputs are set to 0 and the rotor is only exited by initial unbalances.

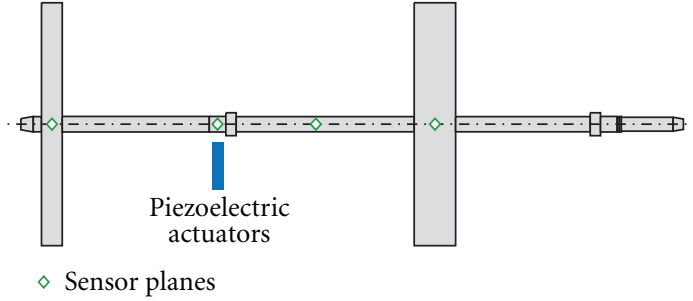

Figure 5: An 8-sensor configuration of the test rig for simulation.

Signals at 5 different rotary frequencies that cover the whole rotary frequency range are simulated. Based on each of the simulated signals in steady state, an unknown input distribution matrix $E_{i}$ with 6 columns is estimated using augmented observer. The $5 E_{i}$ are then combined as a vector set:

$$
E_{M}=\left[E_{1}, E_{2}, \ldots E_{10}\right]
$$

Using the singular value decomposition technique introduced in (15) and (16) a 6-column matrix $E$ is calculated corresponding to the first 6 most significant singular values.

For fault detection a UIO is designed. For the stabilization of the system matrix TA (see (19), (20), and (23)) the pole placement technique is used to determine matrix $K_{1}$ in (23). The pole placement for UIO is problematic in the simulation. The eigenvalues of $T A$ are widely distributed on the complex plane. A pair of eigenvalues of $T A$ (i.e., poles before the stabilization) lie far left on the complex plane (in the order of $-10^{4}$ ), a relocation of these poles causes numerical problems. Thus these poles are kept in the pole placement process. The other poles are placed in a region that is 10 times further left than the poles of the original system.

\subsection{Consideration of Gyroscopic Effect as Model Uncertainties.} Dependent on the available information about the system, the physical model (6a) and (6b) or identified model (7a) and (7b) at different rotary frequencies can be used for this approach. In this example, the physical model is used. A 2column matrix $E$ is estimated using the same method as in Section 4.1. Since the gyroscopic effect is already included in the system matrices, this estimate of $E$ consists only the influences of initial unbalances. Thus a 2-column E-matrix is enough for the representation.

To constrain the observer eigenvalues to a specified region in the left complex half-plane, we add these two following additional LMIs to optimization problem (38a), (38b), and (38c)

$$
\begin{gathered}
\operatorname{He}\left(P_{11} A_{n}-X C\right)+2 \delta P_{11} \prec 0, \\
{\left[\begin{array}{cc}
-r P_{11} & P_{11} A_{n}-X C \\
\star & -r P_{11}
\end{array}\right] \prec 0 .}
\end{gathered}
$$

While (41a) ensures that all real parts of the observer eigenvalues are smaller than $-\delta$, (41b) guarantees that the eigenvalues lie in a circular region around the origin with radius $r$ [42]. To ensure a sufficient detection speed we 

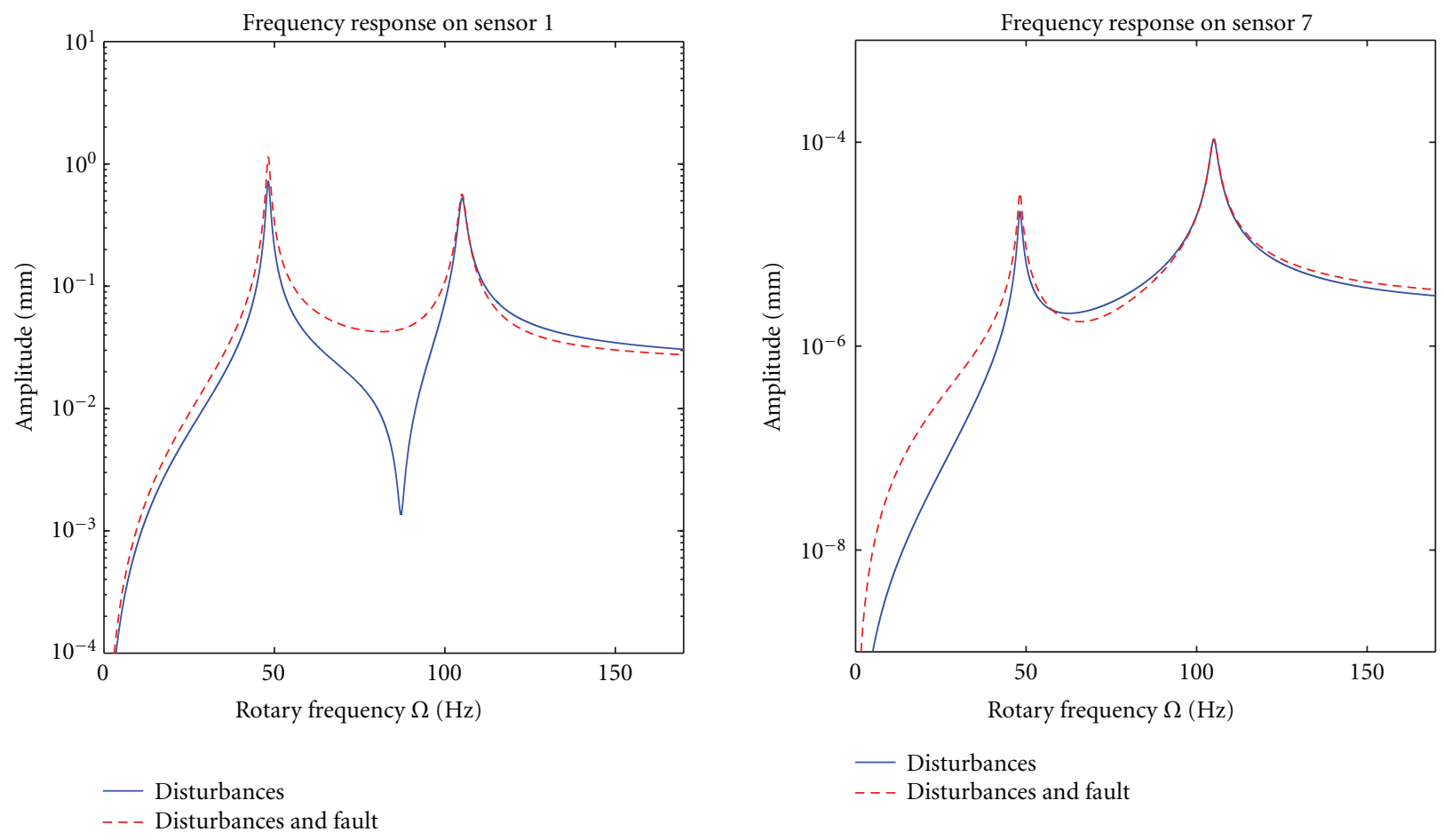

FIGURE 6: Frequency response of disturbances and fault on sensor 1 and sensor 7.
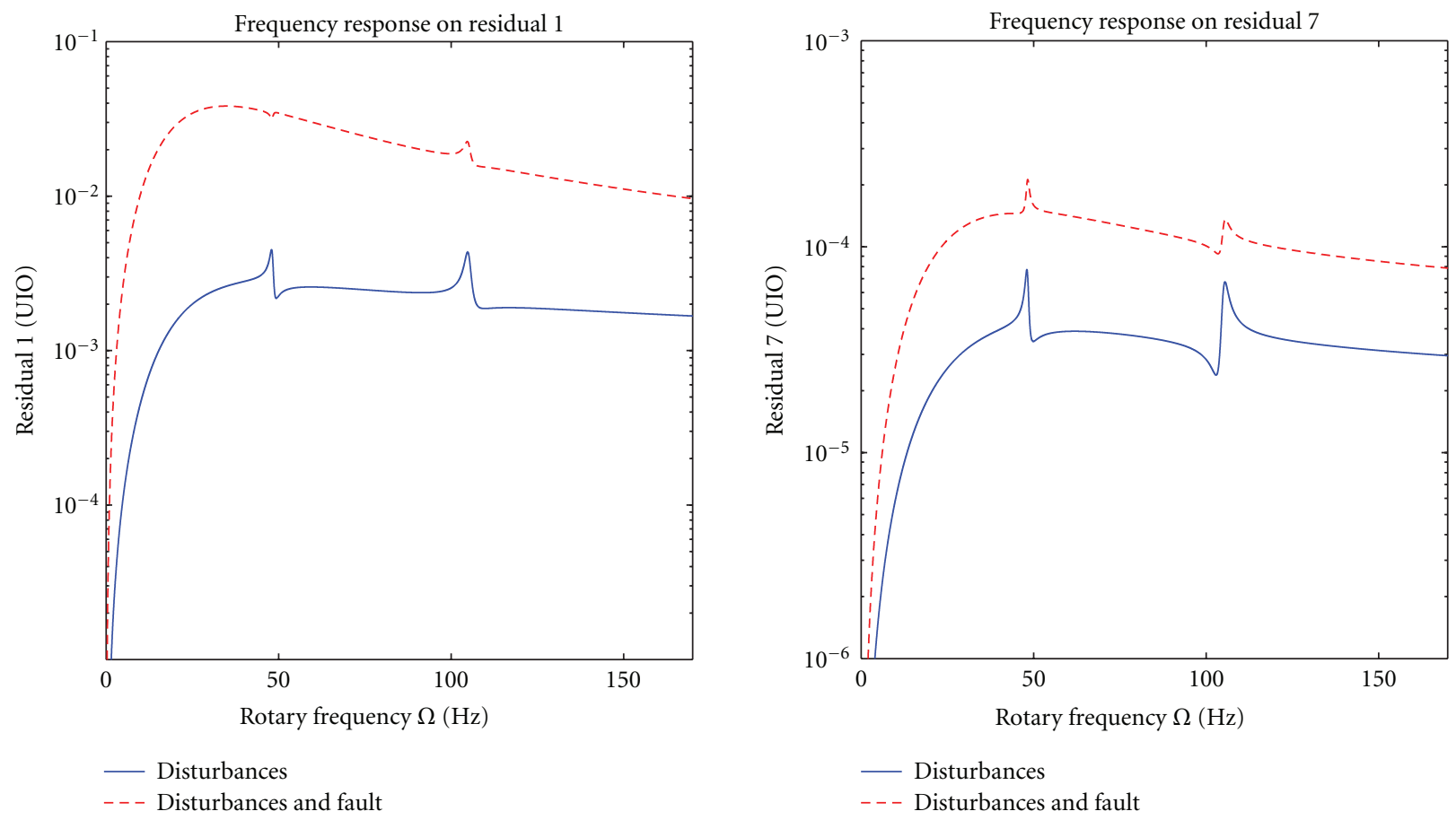

FigURE 7: Frequency response of disturbances and fault on the residuals generated by unknown input observer.

select $\delta=15$ and prevent overly large absolute values of the observer eigenvalues by $r=10^{5}$. Both values $(\delta$ and $r$ ) have been found to give good detection performance in the simulations and tests with measurement data.
4.3. Simulation and Results. The fault is implemented as an additional unbalance in point form which is as large as the initial unbalance on the left disc and is placed orthogonal to the initial unbalance on the disc. Thus the resulted unbalance 

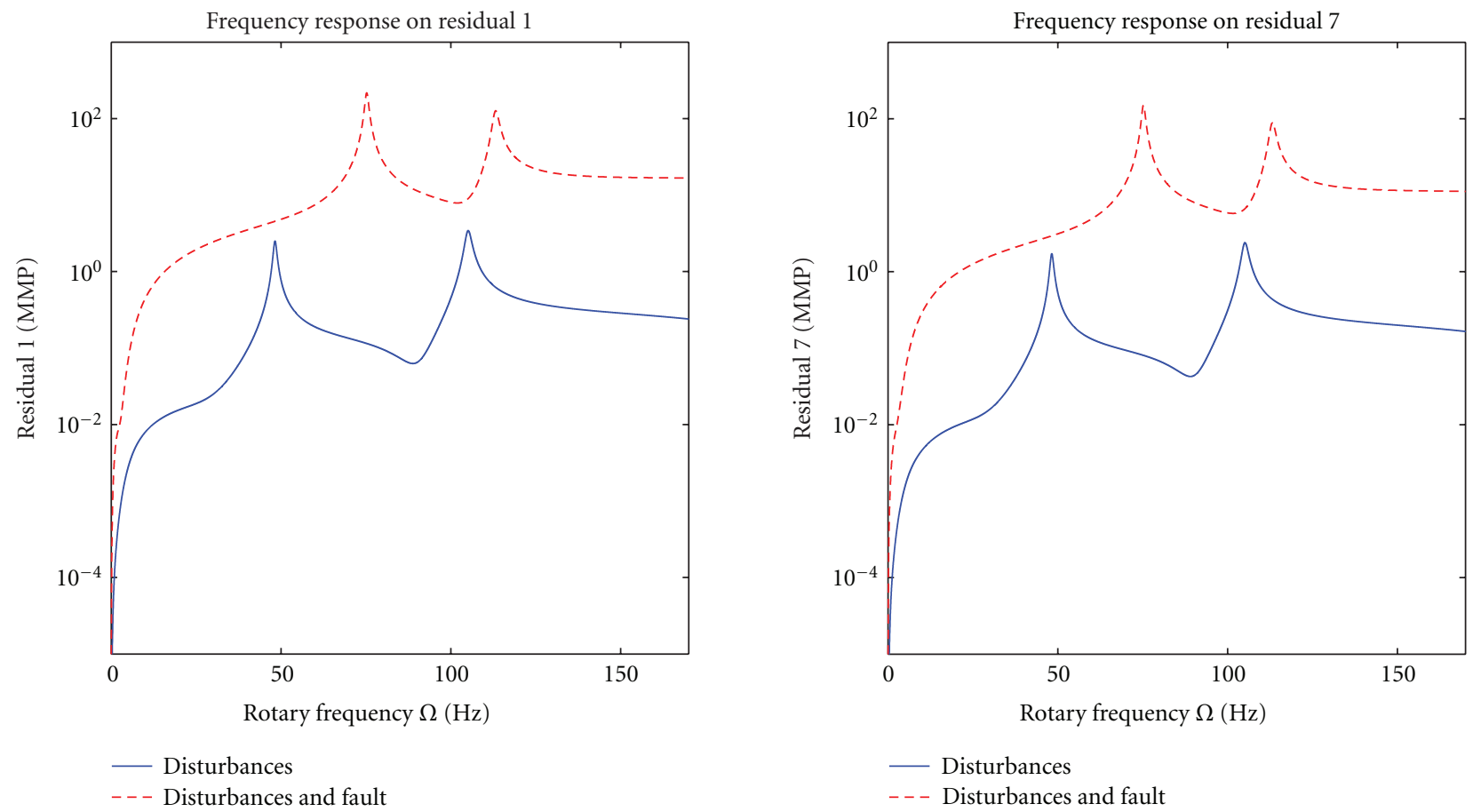

FIGURE 8: Frequency response of disturbances and fault on the residuals generated by the observer designed by model matching.
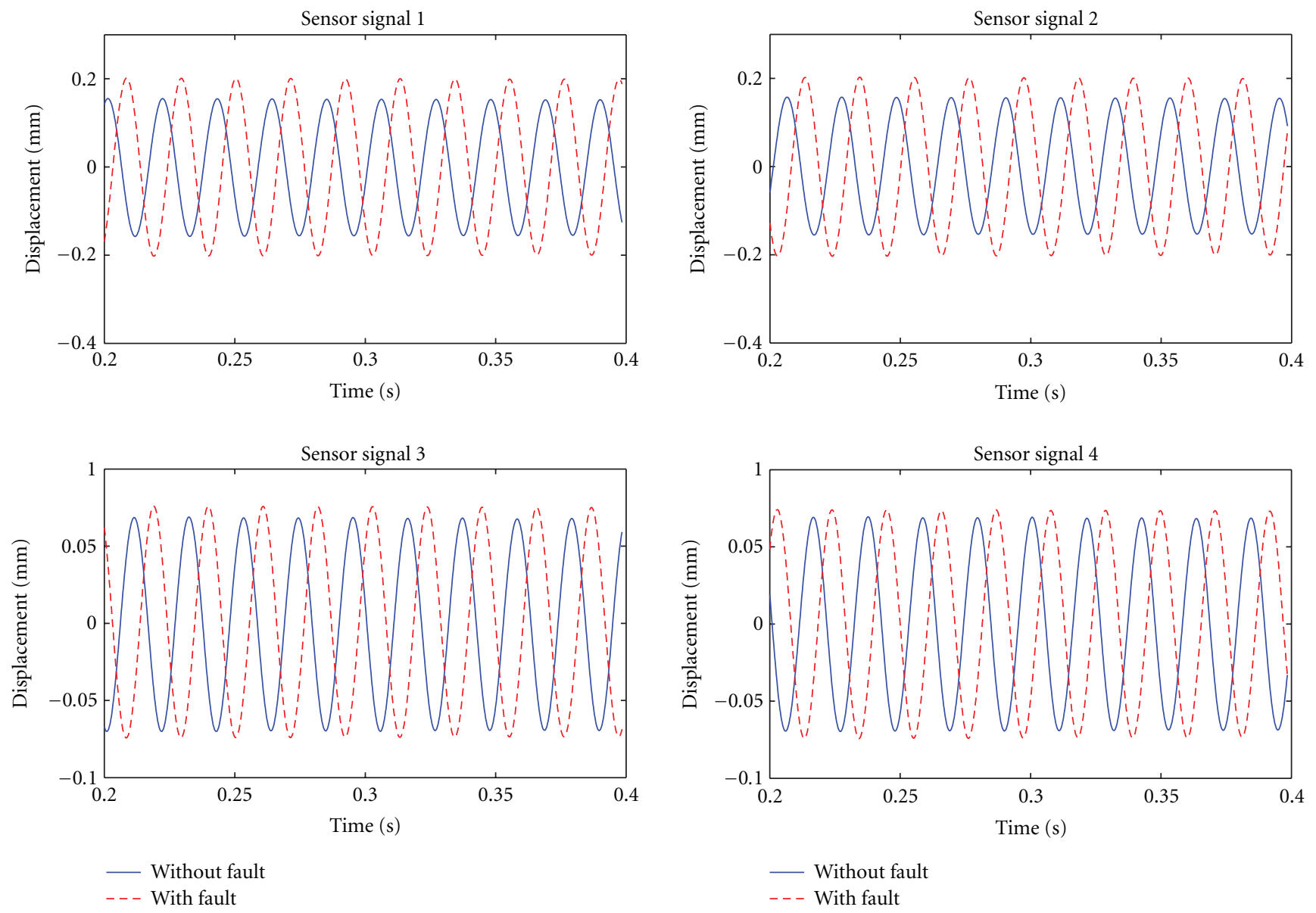

FIGURE 9: Measurements in steady states at rotary frequency of $47 \mathrm{~Hz}$. 

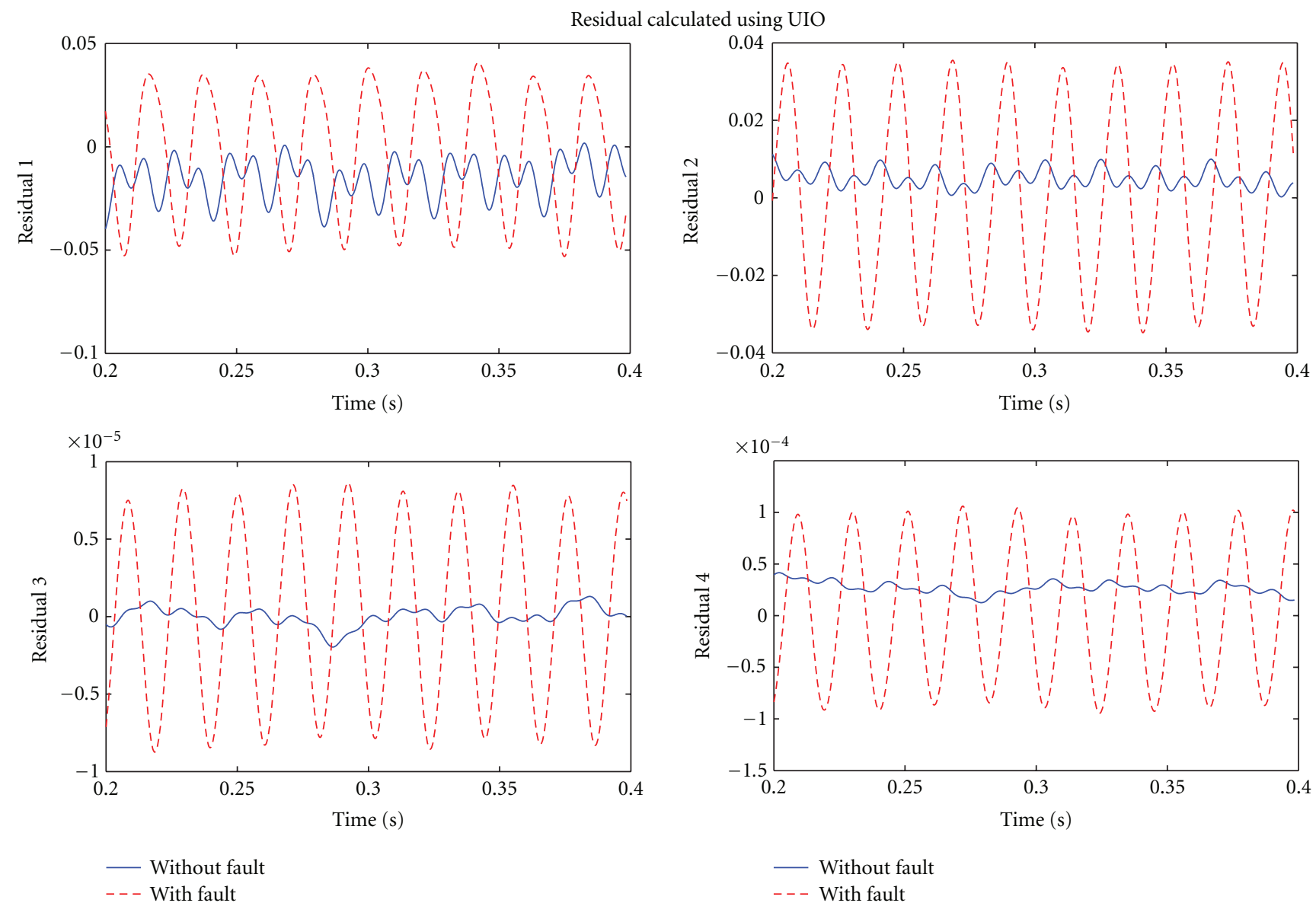

FIgURE 10: Residual generated by UIO.

change is about $41 \%$ in the absolute value on the left disc. Under the influences of the unbalances on the other part of the rotor system, the influences of the fault are almost invisible on the output signals compared to the fault free case. The frequency response on the output signals in fault free case (i.e., exited only by initial unbalances) and in case of fault (i.e., exited by initial unbalances and the additional unbalance on the disc) are presented in Figure 6. Sensor 1 measures the oscillation of the rotor disc on the left side and sensor 7 measures the oscillation of the rotor disc in the middle of the test rig (see Figure 5). The sensors 1 and 7 are suitable to measure the first and second eigenforms (see Figure 3 ).

The result of the unknown input observer is presented in Figure 7. It is observed that although the frequency responses vary for the different sensor outputs, the residuals generated by the UIO have a similar shape. The frequency response on the residual in case of fault is in general much stronger than in the fault free case. For the whole rotary frequency range, the fault detection is based on the rotor model at $\Omega=0$; it proves that the gyroscopic effect can be considered as unknown inputs in the FDI processes.

The residuals generated by observer designed by solving the model matching problem (MMP) are presented in Figure 8 . Also in this case, the fault can be easily detected by inspecting the residuals. It can be observed that the dynamics of the system employing the observer is different from the original system and the resonances in fault free case and in case of fault appears at different frequencies. Thus at the resonances in fault free case and at the anti-resonances in case of fault the result is not as good as in other frequency range. As mentioned in Section 3.3, this might be due to the conservatism introduced to convexify the optimization problem (34). Iterative solutions of the underlying bilinear matrix inequality (BMI) problem might improve the results but are difficult to solve because they are non-convex.

\section{Application on Rotor Test Rig}

To test the methods on the rotor test rig, we apply an additional unbalance of $14.3 \mathrm{gmm}$ on the left rotor disc as fault. The influences of initial unbalances are considered as major disturbances. We use low-pass filters with cutoff frequency of $300 \mathrm{~Hz}$ to attenuate high frequent noise. Figure 9 shows the influences of the fault on the sensor outputs. The changes in the amplitudes caused by the fault are relatively small. The fault causes rather changes in the phases.

As mentioned in Section 4 we need 6 sensors to represent the gyroscopic effect using unknown inputs for whole rotary frequency range. In order to apply UIO we focus on fault 

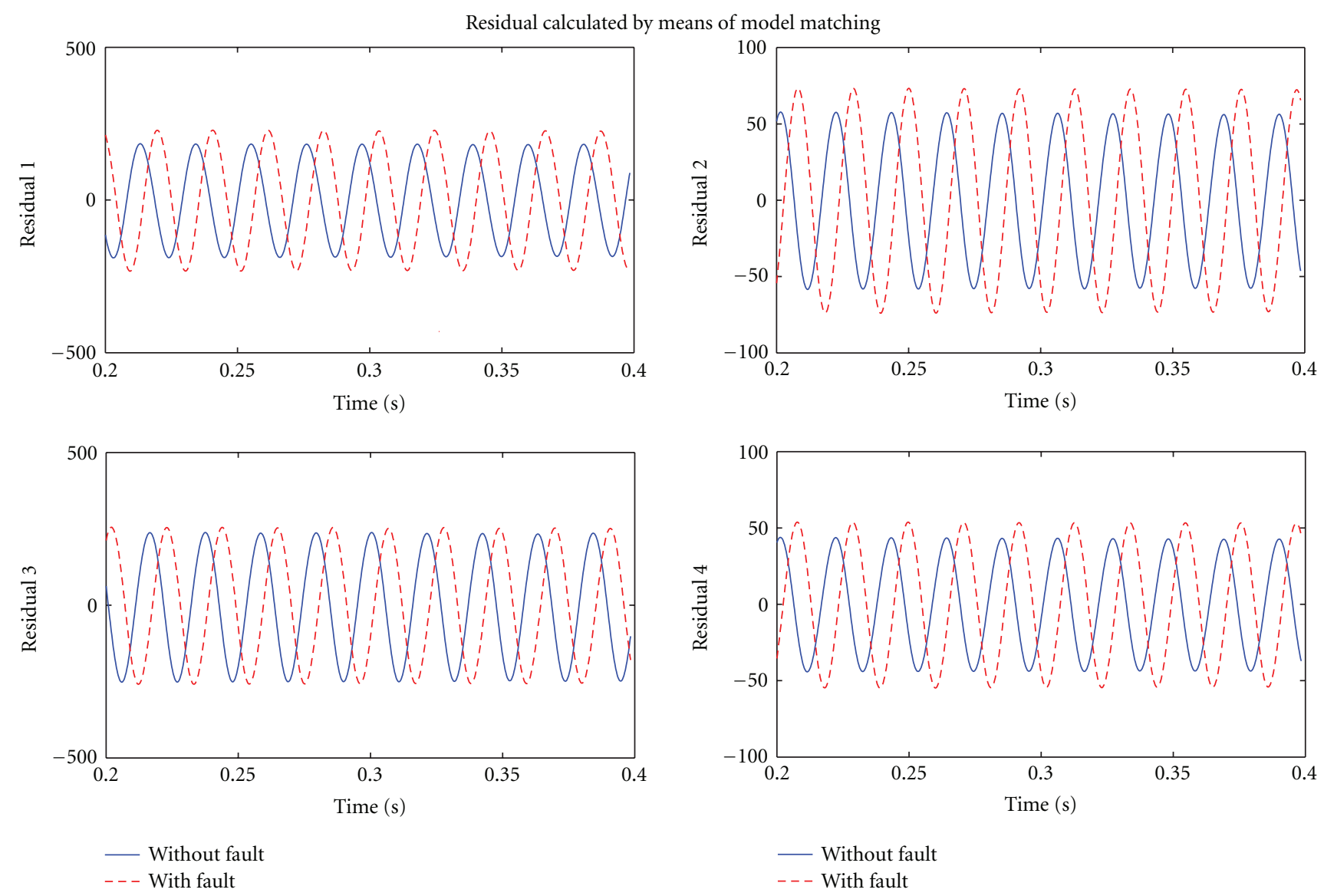

FIGURE 11: Residual generated by means of model matching.

detection at the first resonance excited by unbalances. On the basis of the model of non-rotating rotor a 2-column $E$-matrix is estimated to represent the influences of initial unbalances and gyroscopic effect using the measurements at a rotary frequency of $47 \mathrm{~Hz}$. Since only measurements from 4 sensor are available, only the first 4 eigenfrequencies ( 2 for forward whorl and 2 for backward whorl) are considered in the estimation. The influences of higher modal are neglected. Since the modals with high eigenfrequencies are not strongly excited in the measurement, we assume that the estimation is accurate enough for the application of UIO. For the application of UIO the pole placement process is not problematic in contrary to the case of simulation. All the poles are places in the same region that is 10 times further left than the poles of the original system.

The Results are presented in Figure 10. It can be seen that the UIO successfully suppress the influences of disturbances and model uncertainties on the residuals. The influences of the fault on the residuals are obvious and can be easily detected.

For the application of the method based on solving MMP, we use the physical model (2a) and (2b) with modeled gyroscopic matrix to design the residual generator. We use the rotor model at $47 \mathrm{~Hz}$ for the estimation of the influences of initial unbalances (i.e., disturbances). Since the gyroscopic is already considered in the model, this estimate only represents the disturbances. The design process is the same as in the simulation.

The generated residuals are presented in Figure 11. In this case the optimization method does not work well. Comparing the residuals in case of fault with the residuals in fault free case Figure 11 only shows slightly enhanced differences in percentage terms. In the application we try to set the model in the dear of first resonance as nominal model. But the result is not improved significantly. Although there is no strong restriction of sensor number for the application of optimization methods, the FDI results are strongly influenced by the number of measurements. In the model matching process the considered model uncertainties caused by the gyroscopic effect consists changes in all modals in contrary to the UIO case. That might be too much for optimization with only 4 sensors. Also the accuracy of the physical model might also be a reason for the results. We only considered the gyroscopic effect as model uncertainty, but the model inaccuracies caused by other unmodeled dynamics are not considered for model matching. In case of UIO the model inaccuracies are considered as part of the unknown inputs in the estimation of their distribution matrix by means of measurements. Thus using identified model for FDI might also improve the result of model matching. 


\section{Conclusion}

Two approaches to consider the gyroscopic effect (i.e., as unknown inputs or as model uncertainties) in the model based FDI processes of rotor systems are presented in this paper. Different models (i.e., physical model and identified model) are discussed. Both of the approaches can be applied on the basis of both physical model and identified model. FDI methods with constant parameters can be designed on the basis of these approaches, which is advantageous over the rotary frequency dependent FDI methods.

Robust fault detection against unknown inputs by means of UIO and robust fault detection against model uncertainties by means of model matching are presented. For the estimation of unknown input distribution matrix 2 methods, that is, de-convolution method and augmented observer, are introduced. As an example for fault detection, an additional unbalance on the rotor disc is considered as fault. The feasibility tests of UIO and model matching are carried out by means of simulation. Both methods show good results in the simulation with 8 sensors for whole rotary frequency range, which proves the feasibilities of these approaches if enough measurements are available. To test the methods on the rotor test rig with 4 sensors, fault detections are performed at the first resonance excited by the unbalances. While the UIO still provides a very good result, the FDI performance on the basis of model matching is not satisfactory. The possible reasons are discussed.

For the future work, we are going to integrate more sensors in the test rig in order to test the methods for whole rotary frequency range. The influences of the sensor number on the FDI performance using model matching are to be investigated.

\section{Acknowledgment}

This work was supported by the German Research Foundation (DFG) within the Graduate College GRK1344 in cooperation with Rolls-Royce Deutschland Ltd. \& Co. KG, and the Deutsche Telekom Stiftung.

\section{References}

[1] R. Isermann, Fault-Diagnosis Systems, Springer, Berlin, Germany, 2006.

[2] T. Kreuzinger-Janik and H. Irretier, "Experimental modal analysis-a tool for unbalance identification of rotating machines," International Journal of Rotating Machinery, vol. 6, no. 1, pp. 11-18, 2000.

[3] A. W. Lees, J. K. Sinha, and M. I. Friswell, "The identification of the unbalance of a flexible rotating machine from a single rundown," Journal of Engineering for Gas Turbines and Power, vol. 126, no. 2, pp. 416-421, 2004.

[4] P. G. Morton, "Modal balancing of flexible shafts without trial weights," Proceedings of the Institution of Mechanical Engineers, vol. 199, no. 1, pp. 71-78, 1985.

[5] A. El-Shafei, A. S. El-Kabbany, and A. A. Younan, "Rotor balancing without trial weights," The ASME Journal of Engineering for Gas Turbines and Power, vol. 126, pp. 604-609, 2004.
[6] M. Friswell, J. Penny, S. Garvey, and A. Lees, Dynamics of Rotating Machines, Cambridge University Press, New York, NY, USA, 2010.

[7] A. A. Younan and A. El-Shafei, "Model calibration of anisotropic rotordynamic systems with speed-dependent parameters," ASME Journal of Engineering for Gas Turbines and Power, vol. 130, no. 4, Article ID 042502, 10 pages, 2008.

[8] J. Chen and R. J. Patton, Robust Model-Based Fault Diagnosis for Dynamic Systems, Kluwer Academic Publishers, Dordrecht, The Netherland, 1999.

[9] S. X. Ding, Model-Based Fault Diagnosis Techniques, Springer, Berlin, Germany, 2008.

[10] K. Watanabe and D. M. Himmelblau, "Instrument fault detection in systems with uncertainties," International Journal of Systems Science, vol. 13, no. 2, pp. 137-158, 1982.

[11] P. Frank and J. Wünnenberg, "Robust fault diagnosis using unknown input schemes," in Fault Diagnosis in Dynamic Systems: Theory and Application, pp. 47-98, Prentice Hall, Upper Saddle River, NJ, USA, 1989.

[12] R. J. Patton and J. Chen, "Uncertainty modeling and robust fault diagnosis for dynamic systems," in Issues of Fault Diagnosis for Dynamic Systems, pp. 189-209, Springer, Berlin, Germany, 2000.

[13] R. J. Patton and J. Chen, "Robust fault detection using eigenstructure assignment: a tutorial consideration and some new results," in Proceedings of the 30th IEEE Conference on Decision and Control, pp. 2242-2247, Brighton, UK, December 1991.

[14] R. J. Patton and J. Chen, "A robust parity space approach to fault diagnosis based on optimal eigenstructure assignment," in Proceedings of the IEE International Control Conference (Control '91), pp. 1056-1061, Peregrinus Press, Edinburgh, UK, March 1991.

[15] A. Varga, "On designing least order residual generators for fault detection and isolation," in Proceedings of 16th International Conference on Control Systems and Computer Science, pp. 323-330, Bucharest, Romania, 2007.

[16] A. Varga, "On computing nullspace bases-a fault detection perspective," in Proceedings of the International Federation of Automatic Control (IFAC '08), pp. 6295-6300, Seoul, Korea, 2008.

[17] Z. Wang, R. S. Schittenhelm, and S. Rinderknecht, "Augmented observer for fault detection and isolation (FDI) in rotor systems," in Proceedings of the World Congress on Engineering and Computer Science (WCECS '12), San Francisco, Calif, USA, October 2012.

[18] S. Boyd, L. E. Ghaoui, E. Feron, and V. Balakrishnan, Linear Matrix Inequalities in System and Control Theory, SIAM, Philadelphia, Pa, USA, 1994.

[19] H. B. Wang, J. L. Wang, and J. Lam, "Robust fault detection observer design: iterative LMI approaches," Transactions of the ASME: Journal of Dynamic System Measurement \& Control, vol. 129, no. 1, pp. 77-82, 2007.

[20] H. Wang, H.-H. Ju, and G.-H. Yang, "Fault detection filter design for linear polytopic uncertain continuous-time systems," Acta Automatica Sinica, vol. 36, no. 5, pp. 742-750, 2010.

[21] P. M. Frank and X. Ding, "Frequency domain approach to optimally robust residual generation and evaluation for model-based fault diagnosis," Automatica, vol. 30, no. 5, pp. 789-804, 1994.

[22] D. Sauter and F. Hamelin, "Frequency-domain optimization for robust fault detection and isolation in dynamic systems," 
IEEE Transactions on Automatic Control, vol. 44, no. 4, pp. 878-882, 1999.

[23] E. Mazars, I. M. Jaimoukha, Z. Li, and A. C. Zolotas, "Fault detection and isolation filter design for systems subject to polytopic uncertainties," in Proceedings of the 15th IEEE Mediterranean Conference on Control and Automation (MED '07), pp. 1-5, Athens, Ga, USA, June 2007.

[24] M. Zhong, S. X. Ding, J. Lam, and H. Wang, "An LMI approach to design robust fault detection filter for uncertain LTI systems," Automatica, vol. 39, no. 3, pp. 543-550, 2003.

[25] A. S. Sekhar and B. S. Prabhu, "Effects of coupling misalignment on vibrations of rotating machinery," Journal of Sound and Vibration, vol. 185, no. 4, pp. 655-671, 1995.

[26] A. W. Lees, "Misalignment in rigidly coupled rotors," Journal of Sound and Vibration, vol. 305, no. 1-2, pp. 261-271, 2007.

[27] K. P. Gertzos, P. G. Nikolakopoulos, A. C. Chasalevris, and C. A. Papadopoulos, "Wear identification in rotor-bearing systems by measurements of dynamic bearing characteristics," Computers and Structures, vol. 89, pp. 55-66, 2011.

[28] S. Seibold and K. Weinert, "A time domain method for the localization of cracks in rotors," Journal of Sound and Vibration, vol. 195, no. 1, pp. 57-73, 1996.

[29] A. Eduardo and R. Pederiva, "Fault diagnosis in stationary rotor systems through correlation analysis and artificial neural network," in Proceedings of the 17th International Congress of Mechanical Engineering, pp. 1-9, 2003.

[30] B. Hasch, O. Lindenborn, and R. Nordmann, "Model-based fault detection on a rotor in an actively supported bearing using piezoelectric actuators and the fxlms-algorithm," in Proceedings of the Motion and Vibration Control (MOVIC '08), September 2008.

[31] G. Genta, Dynamics of Rotating Systems, Springer, New York, NY, USA, 2005.

[32] R. S. Schittenhelm, Z. Wang, and S. Rinderknecht, "Observer design for rotating shafts excited by unbalance," in Proceedings of the 10th International Conference on Vibrations in Rotation Machinery, London, UK, 2012.

[33] Z. Wang, R. S. Schittenhelm, and S. Rinderknecht, “Observer design for unbalance excited rotor systems with gyroscopic effect," in Proceedings of the IEEE International Conference on Mechatronics and Automation (ICMA '12), Sichuan, China, August 2012.

[34] R. J. Patton, J. Chen, and H. Y. Zhang, "Modelling methods for improving robustness in fault diagnosis of jet engine system," in Proceedings of the 31st IEEE Conference on Decision and Control, pp. 2330-2335, Tucson, Ariz, USA, 1992.

[35] R. J. Patton, H. Y. Zhang, and J. Chen, "Modelling of uncertainties for robust fault diagnosis," in Proceedings of the 31st IEEE Conference on Decision and Control, pp. 921-926, Tucson, Ariz, USA, 1992.

[36] R. J. Patton and J. Chen, "Optimal unknown input distribution matrix selection in robust fault diagnosis," Automatica, vol. 29, no. 4, pp. 837-841, 1993.

[37] E. Frisk and L. Nielsen, "Robust residual generation for diagnosis including a reference model for residual behavior," Automatica, vol. 42, no. 3, pp. 437-445, 2006.

[38] E. Mazars, I. M. Jaimoukha, and Z. Li, "Computation of a reference model for robust fault detection and isolation residual generation," Journal of Control Science and Engineering, vol. 2008, Article ID 790893, 12 pages, 2008.
[39] S. X. Ding, E. L. Ding, and T. Jeinsch, "A new optimization approach to the design of fault detection filters," in Proceedings of the International Federation of Automatic Control (IFAC) Symposium SAFEPROCESS: Fault Detection, Supervision and Safety for Technical Processes (SAFEPROCESS 'O0), pp. 250255, Budapest, Hungary, 2000.

[40] E. Ostertag, "An improved path-following method for mixed $\mathrm{H}_{2} / \mathrm{H}_{\infty}$ controller design," IEEE Transactions on Automatic Control, vol. 53, no. 8, pp. 1967-1971, 2008.

[41] A. Wahrburg, S. Khodaverdian, and J. Adamy, "Robust observer-based fault detection and isolation in the standard control problem framework," in Proceedings of the 7th IFAC Symposium on Robust Control Design, Aalborg, Denmark, July 2012.

[42] M. Chilali, P. Gahinet, and P. Apkarian, "Robust pole placement in LMI regions," IEEE Transactions on Automatic Control, vol. 44, no. 12, pp. 2257-2270, 1999. 

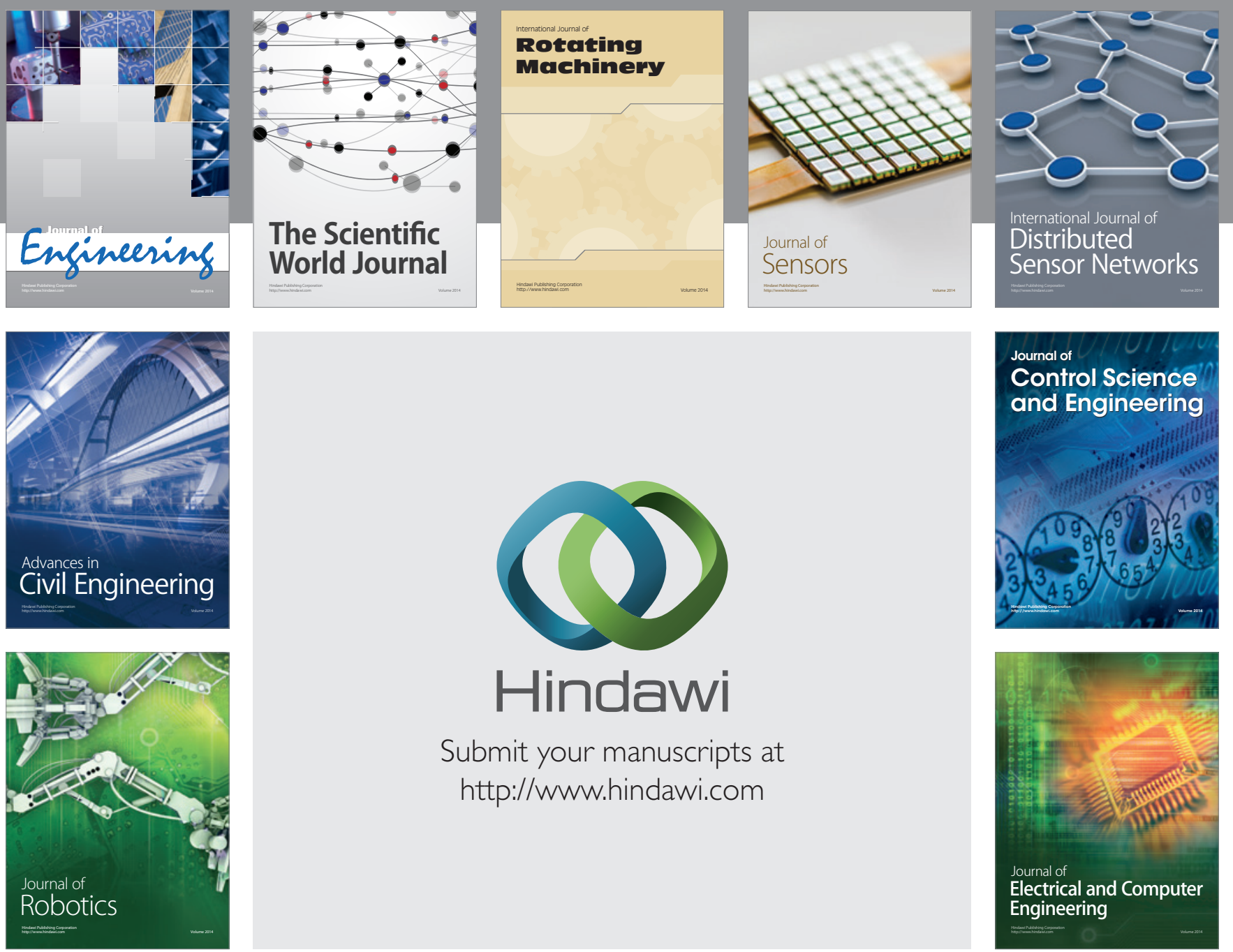

Submit your manuscripts at

http://www.hindawi.com
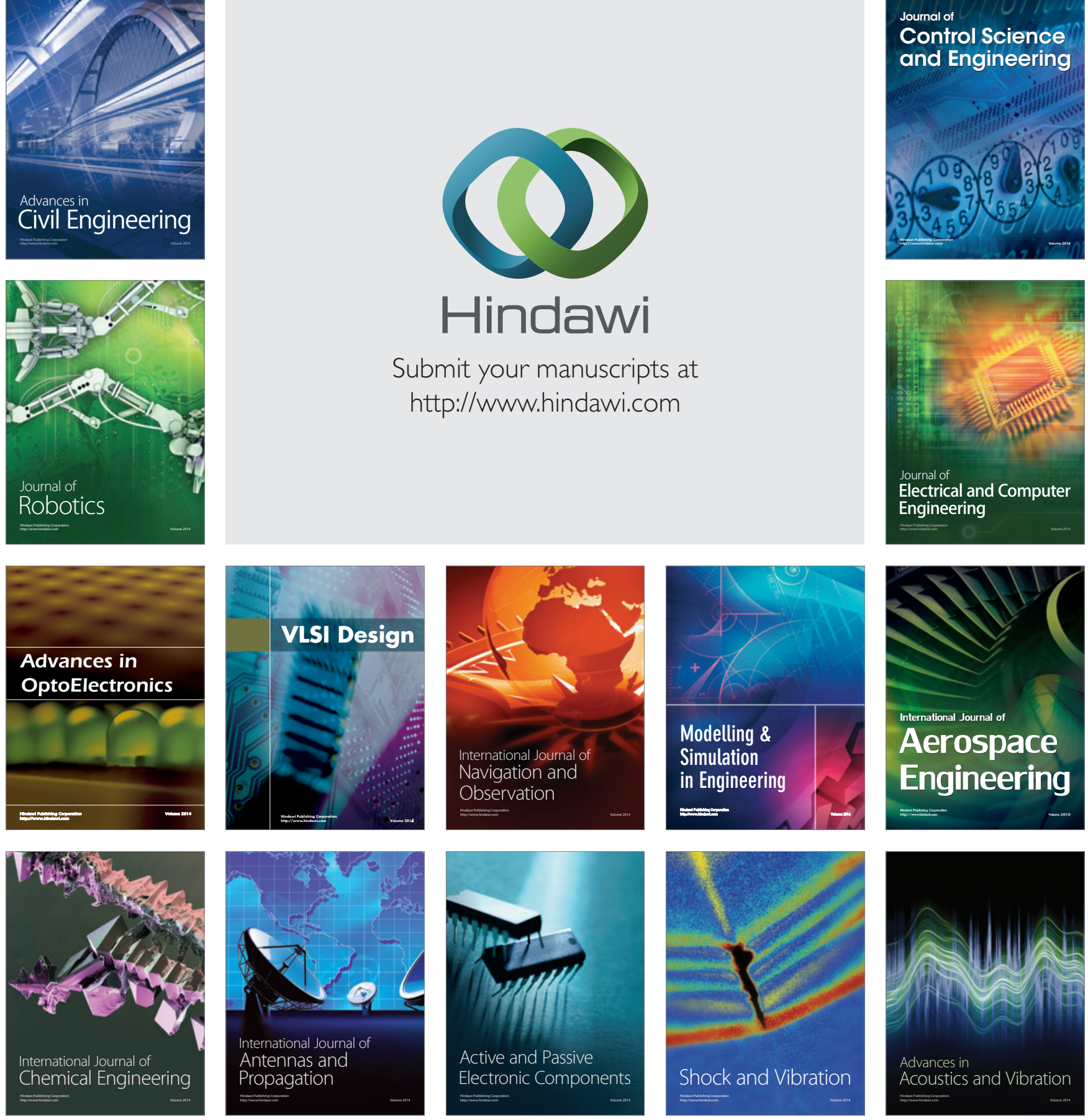